\title{
Heisenberg chain equations in terms of Fockian covariance with electric field account and multiferroics in nanoscale
}

\author{
S. Leble \\ Baltic Federal University, st. A.Nevskogo, 14, Kaliningrad, Russia, 236006 \\ lebleu@mail.ru
}

DOI 10.17586/2220-8054-2019-10-1-18-30

\begin{abstract}
Heitler-Heisenberg multispin states were studied via irreducible representations of the united symmetry with respect to permutations and space transformations group. The mean energy is given in explicit form in terms of the characters of the joint group irreducible representations. The system's Fockian covariance incorporates its exchange integral of the self-consistent states into the Heisenberg chain theory. External fields account is delivered in perturbation theory frame. Its application to statistical physics approach leads to the thermodynamic parameter evaluation. The nanotube example with space symmetry including rotations and translations, is studied. Its symmetry introduces basic closest neighbor exchange integrals that enter the statistical sum.
\end{abstract}

Keywords: multielectron states, permutation-space symmetry, mean energy, Gauss distribution, Heisenberg chain, Fockian symmetry, electric field, nanotube, multiferroics.

Received: 24 January 2019

Revised: 10 February 2019

\section{Introduction}

A quantum theory of a multielectron system bases on joint symmetry group of permutations and space symmetry. In the pioneer paper of Heisenberg [1] it was established, that Weiss model of ferro-magnetism is explained by electric interaction, which origin may be understood on base of theory of Heitler-London hydrogen molecule. It is naturally combined with Hartree-Fock theory [2] and its further self-consistent one-particle generalizations that provide better exchange integral evaluation including the system spectral properties [3].

The group theoretical foundation of [1] is based on multispin state via irredicible representations of the symmetry with respect to permutation group [4]. The problem of Hamiltonian spectrum parameters evaluation is expressed in terms of irreducible representations of symmetry group and exchange integrals, see also [5].

The next important step in the theory development taking into account the antisymmetry of the wave function of electrons and a more advanced extended symmetry, including space (geometry) symmetry of equivalent positions of atoms equilibrium. The magnetic properties of matter exist as a result of electrons spins and the antisymmetry of the wave function.

When a system is in an external electric or magnetic field, an additional terms in the Hamiltonian appear. Its microscopic origin is conventionally understand in terms of perturbation theory that gives deformation of wavefunctions and energy eigenvalues. The results in atomic physics are known as Stark and Zeeman effects.

A multiferroic control implies a manipulation of magnetic moment by electric field switching. General thermodynamic consideration, specified by Dzialoshinski, introduces the magnetoelectric constants, namely longitudinal and perpendicular dielectric constants, the magnetic susceptibilities, and the constants describing the magnetoelectrical effect.

In this paper, a spectral and statistical theory in the Heisenberg spirit is developed. The application of statistical physics approach, formulated in [1] leads to the mentioned thermodynamic parameters evaluation with external magnetic and electric fields account. The ferro-magnetism appearance conditions are illustrated by plotting the dependence of closest neighbors number on exchange integral.

Applications to amorphous microwires and crystalline nanowires are discussed by example of a tube made of atoms which space positions form symmetrical configuration. The energy and distribution parameter modifications for nanotubes naturally introduce the closest neighbors exchange integrals defined by the nanostructure symmetry. It gives a model thermodynamic description based on a statistical sum modifications. 


\section{A joint symmetry group of multi-electron systems}

Generally a wave function of a multi-electron system:

$$
\Phi=\Phi\left(\vec{R}_{1}, \cdots, \vec{R}_{K},\left(\vec{r}_{1}, \sigma_{1}\right), \cdots,\left(\vec{r}_{N}, \sigma_{N}\right)\right) \in \mathcal{H}
$$

depends on the variables $\vec{R}_{j} \in R^{3}, j=1, \ldots K$, as on parameters, that are coordinates of "adiabatic" atoms positions, $\vec{r}_{i} \in R^{3}, i=1, \ldots, N$ are electron coordinates and $\sigma_{1}, \sigma_{2}, \ldots, \sigma_{N}$ are spin variables of electrons. Since the electrons are fermions, the Pauli-Luders theorem states that the wave function must be antisymmetric in the variables of the electrons; this means that the function belongs the irreducible representation of the all-electrons permutation group.

A rotation in position space marked as $R(\alpha, \beta, \gamma)$, where the angles $\alpha, \beta, \gamma$ are Euler ones represents in a 1 -electron spin space by a $2 \times 2$ matrix $u(\alpha, \beta, \gamma)$ :

$$
u(\alpha, \beta, \gamma)=\mathcal{D}^{1 / 2}(\alpha, \beta, \gamma)
$$

where $D^{1 / 2}$ is an unitary matrix of irreducible representation of rotation group [6]. This means that the wave function (1) transforms as direct product of the representations (2). To study the joint group table it is convenient to "jump" into the representation of the spinors $\Phi$, because the permutations and rotations act also to the atoms positions. The actions in Hilbert space of states are for the permutations:

$$
\begin{gathered}
\mathcal{P} \Phi\left(\vec{R}_{1}, \cdots, \vec{R}_{K},\left(\vec{r}_{1}, \sigma_{1}\right), \cdots,\left(\vec{r}_{N}, \sigma_{N}\right)\right)= \\
\Phi\left(\vec{R}_{1}, \cdots, \vec{R}_{K},\left(P \vec{r}_{1}, P \sigma_{1}\right), \cdots,\left(P \vec{r}_{N}, P \sigma_{N}\right)\right)
\end{gathered}
$$

and

$$
\begin{gathered}
\mathcal{R} \Phi\left(\vec{R}_{1}, \cdots, \vec{R}_{K},\left(\vec{r}_{1}, \sigma_{1}\right), \cdots,\left(\vec{r}_{N}, \sigma_{N}\right)\right)= \\
\mathcal{D}_{\sigma_{1}, \sigma_{1}^{\prime}}^{1 / 2} \ldots \mathcal{D}_{\sigma_{N}, \sigma_{N}^{\prime}}^{1 / 2} \Phi\left(\vec{R}_{1}, \cdots, \vec{R}_{K},\left(R \vec{r}_{1}, \sigma_{1}^{\prime}\right), \cdots,\left(R \vec{r}_{N}, \sigma_{N}^{\prime}\right)\right)
\end{gathered}
$$

for rotations. The operators $\mathcal{P}, \mathcal{R}$ act in Hilbert space $\mathcal{H}$, while $P, R$ act in position space $R^{3}$.

A combination of the transformations (4) and (6):

$$
\begin{gathered}
\mathcal{P} \mathcal{R} \Phi\left(\vec{R}_{1}, \cdots, \vec{R}_{K},\left(\vec{r}_{1}, \sigma_{1}\right), \cdots,\left(\vec{r}_{N}, \sigma_{N}\right)\right)= \\
\mathcal{D}_{P \sigma_{1}, \sigma_{1}^{\prime}}^{1 / 2} \mathcal{D}_{P \sigma_{N}, \sigma_{N}^{\prime}}^{1 / 2} \Phi\left(\vec{R}_{1}, \cdots, \vec{R}_{K},\left(P R \vec{r}_{1}, \sigma_{1}^{\prime}\right), \cdots,\left(P R \vec{r}_{N}, \sigma_{N}^{\prime}\right)\right),
\end{gathered}
$$

and, in opposite order,

$$
\begin{gathered}
\mathcal{R P} \Phi\left(\vec{R}_{1}, \cdots, \vec{R}_{K},\left(\vec{r}_{1}, \sigma_{1}\right), \cdots,\left(\vec{r}_{N}, \sigma_{N}\right)\right)= \\
\mathcal{D}_{\sigma_{1}, P \sigma_{1}}^{1 / 2} \ldots \mathcal{D}_{\sigma_{N}, P \sigma_{N}}^{1 / 2} \Phi\left(\vec{R}_{1}, \cdots, \vec{R}_{K},\left(P \vec{r}_{1}, P \sigma_{1}\right), \cdots,\left(R P \vec{r}_{N}, R P \sigma_{N}\right)\right) .
\end{gathered}
$$

For the joint symmetry applications the Frobenius theorem is used. Let $H$ be a subgroup of the group $G$ and $\gamma^{(i)}$ is a irreducible representation of $H$. Then the representation $\Gamma$ of $G$, induced by $\gamma^{(i)}$ contains each irreducible representation $\Gamma^{\lambda}$ of $G$ so many times as $\gamma^{(i)}$ contains in the representation of $H$ given by matrices $\Gamma^{\lambda}$.

The characters of the symmetry group is evaluated by the formula from [7],

Let us give more details for simplest example of Hydrogen molecule. In the case of two-electron system the wave function is defined in six-dimensional position space with the coordinate set $\left\{x_{1}, y_{1}, z_{1} ; x_{2}, y_{2}, z_{2}\right\}$ and with extra discrete spin variables $\sigma_{1}, \sigma_{2}$, that runs numbers $-1,1 . \Phi=\Phi\left(\vec{R}_{1}, \vec{R}_{2} ; \vec{r}_{1}, \sigma_{1} ; \vec{r}_{2}, \sigma_{2}\right)$. Suppose atom positions $\vec{R}_{1}, \vec{R}_{2}$ lie in the plane $x y$ symmetrically to origin.

The permutation, in this case, acts in the direct product of space and spin variables as:

$$
P\left\{x_{1}, y_{1}, z_{1} ; x_{2}, y_{2}, z_{2} ; \sigma_{1}, \sigma_{2}\right\}=\left\{x_{2}, y_{2}, z_{2} ; x_{1}, y_{1}, z_{1} ; \sigma_{2}, \sigma_{1}\right\} .
$$

A rotation in $x y$ plane:

$$
R\left\{x_{1}, y_{1}, z_{1} ; x_{2}, y_{2}, z_{2}\right\}=\left\{(\cos \pi) x_{1},(\cos \pi) y_{1}, z_{1} ;(\cos \pi) x_{2},(\cos \pi) y_{2}, z_{2}\right\} .
$$

is accompanied to a transformation in spin space $u(R) \in S U(2)$ so as, generally

$$
u v_{\sigma}=\mathcal{D}_{\nu \sigma}^{1 / 2} v_{\sigma}
$$

The product of the actions is:

$$
\begin{gathered}
P R\left\{x_{1}, y_{1}, z_{1} ; x_{2}, y_{2}, z_{2} ; \sigma_{1}, \sigma_{2}\right\}= \\
P\left\{(\cos \pi) x_{1},(\cos \pi) y_{1}, z_{1} ;(\cos \pi) x_{2},(\cos \pi) y_{2}, z_{2}\right\},
\end{gathered}
$$


and, in inverse order:

$$
\left.R P\left\{x_{1}, y_{1}, z_{1} ; x_{2}, y_{2}, z_{2} ; \sigma_{1}, \sigma_{2}\right\}=R\left\{x_{2}, y_{2}, z_{2} ; x_{1}, y_{1}, z_{1} ; \sigma_{2}, \sigma_{1}\right\}\right\}
$$

with rotation action by $(8)$.

\section{Born-Oppenheimer approximation. Hamiltonian of the system of electrons of a solid}

In the Born-Oppenheimer approximation, in the problem for electrons, the nuclei of a solid are assumed to be static. The Hamiltonian is written for a system of $N$ electrons, in the field of the nuclei:

$$
\hat{H}=-\frac{\hbar^{2}}{2 m} \sum_{i} \Delta_{i}-\sum_{J, i} \frac{Z_{J} e^{2}}{\left|\vec{r}_{i}-\vec{R}_{J}\right|}+\sum_{i, j \neq i} \frac{e^{2}}{\left|\vec{r}_{i}-\vec{r}_{j}\right|} .
$$

In the expression (12), $\sum_{i}$ is a sum over all electrons, $i$ is the electron number; $\sum_{J}$ is a sum over all nuclei, $J$ is nucleus number; $Z_{J}$ is the charge of the nucleus with number $J$, expressed in charges of an electron module $|e|$; $\vec{R}_{J}$ is a position of the nucleus $J, \vec{r}_{i}$ is a radius-vector for the electron number $i$.

The energy eigenvalue problem is formulated in terms of the equation:

$$
\hat{H} \Phi=E \Phi,
$$

where the eigenfinctions $\Phi=\Phi\left(\vec{R}_{1}, \cdots, \vec{R}_{K},\left(\vec{r}_{1}, \sigma_{1}\right), \cdots,\left(\vec{r}_{N}, \sigma_{N}\right)\right)$, are parametrized by the nuclei positions $\vec{R}_{j}, \sigma_{1}, \sigma_{2}, \ldots \sigma_{N}$ are electron spin variables. Since the electrons are fermions, the Pauli principle claims, that a wave function must be antisymmetric in the variables of the electrons, for example,

$$
\Phi\left(\vec{R}_{1}, \cdots, \vec{R}_{K},\left(\vec{r}_{1}, \sigma_{1}\right), \cdots,\left(\vec{r}_{N}, \sigma_{N}\right)\right)=-\Phi\left(\vec{R}_{1}, \cdots, \vec{R}_{K},\left(\vec{r}_{N}, \sigma_{N}\right), \cdots,\left(\vec{r}_{1}, \sigma_{1}\right)\right) .
$$

For shorthand electrons are marked simply with their numbers, that is, we introduce the notation $i=\left(\vec{r}_{i}, \sigma_{i}\right)$ . Then $\Phi=\Phi\left(\vec{R}_{1}, \cdots, \vec{R}_{K}, 1, \cdots, N\right)$, so further the summation by spin variable is implied.

A symmetry of the equation (13) is determined directly from Fock equations.

\section{The Fock equations}

Within the framework of the Hartree-Fock method, the wave function $\Phi=\Phi\left(\vec{R}_{1}, \cdots, \vec{R}_{K}, 1, \cdots, N\right)$ may be searched for in the form of automatically antisymmetric Slater determinant (see $[2,8]$ ) composed of singleparticle functions:

$$
\Phi=\left|\begin{array}{ccccc}
\Psi_{1}(1) & \Psi_{2}(1) & \ldots & \ldots & \Psi_{N}(1) \\
\Psi_{1}(2) & \Psi_{2}(2) & \ldots & \ldots & \Psi_{N}(2) \\
\cdots & \ldots & \ldots & \ldots & \ldots \\
\ldots & \ldots & \ldots & \ldots & \ldots \\
\Psi_{1}(N) & \Psi_{2}(N) & \ldots & \ldots & \Psi_{N}(N)
\end{array}\right|
$$

Here the functions $\Psi_{j}(i)$ are named as spin orbitals. From the variational principle $(\min \langle\Phi, \hat{H} \Phi\rangle)$ the Fock equations for the spin-orbitals $\Psi_{i}$ are derived. Let the set of variables "1,2" be marked as " $x, y$ " as in [9] writing for shorthand:

$$
\int d x=\sum_{\sigma} \int d \vec{r} .
$$

The conventional form of the equations is:

$$
\hat{F}_{j} \Psi_{j}(x)=\varepsilon_{j} \Psi_{j}(x),
$$

and the $\hat{F}_{j}$ are Fockians:

$$
\hat{F}_{j}=\hat{h}_{1}+\sum_{i=1}^{\prime N}\left(\hat{C}_{i}-\hat{J}_{i}\right)
$$

where the Coulombian interaction operator is defined by: .

$$
\hat{C}_{i} \Psi_{j}=\int \frac{\Psi_{i}^{*}(y) \Psi_{i}(y)}{r_{12}} d y \Psi_{j}(x)
$$


where $r_{12}=\left|\vec{r}_{1}-\vec{r}_{2}\right|$ and the exchange one is:

and

$$
\hat{J}_{i} \Psi_{j}=\int \frac{\Psi_{i}^{*}(y) \Psi_{j}(y)}{r_{12}} d y \Psi_{i}(x)
$$

$$
\hat{h}_{1}=-\frac{\hbar^{2}}{2 m} \triangle_{1}-\sum_{J} \frac{Z_{J} e^{2}}{\left|\vec{r}_{1}-\vec{R}_{J}\right|}
$$

A prime over summation sign means that a term with $i=j$ under the sum sign is skipped. The integration is carried out over the variables of particle $2, r_{12}=\left|\vec{r}_{1}-\vec{r}_{2}\right|, \triangle_{1}=\frac{\partial^{2}}{\partial x_{1}}+\frac{\partial^{2}}{\partial y_{1}}+\frac{\partial^{2}}{\partial z_{1}}, \vec{r}_{1}=\left(x_{1}, y_{1}, z_{1}\right)$. It is assumed that the functions $\Psi_{i}$ are orthogonal: $\int \Psi_{i}^{*}(x) \Psi_{k}(x) d x=\delta_{i k}$.

A symmetry of the equation (15) is determined by the following.

\section{Fock equation covariance}

We base on Fock Statement [2]. Let L be a Hermitian operator, which is symmetric with respect to all the electrons, $\Omega=\alpha_{1} \Psi_{1}+\ldots+\alpha_{s} \Psi_{s}$.

$$
A=\frac{\int \bar{\Omega} L \Omega}{\int \bar{\Omega} \Omega}
$$

then the latter does not depend on coefficients $\alpha_{1} ;,,,, \alpha_{s}$. If $\mathrm{H}$ is the energy operator, then, setting the variation of A to zero, one obtains the Schrödinger equation for the function $\Omega$, to infer the Schrödinger equation from the variational principles, for any linear combination of functions. In particular the coefficients may be chosen as matrix elements of irreducible representation $D_{i k}^{\nu}(g)$ of the symmetry group $g \in G$, that defines action:

$$
\Psi_{i}(g x)=\sum_{k}^{s} D_{i k}^{\nu}(g) \Psi_{k}
$$

A symmetry of the equation (13) is determined directly from Fock equations. Application of permutation transformation to the Fock equations system consist of

1) change the order of equations

2) the action of the operator $T_{g}$ on exchange and Coulombian integrals includes action at $r_{12}=|x-y|$, that looks as $T_{g}|x-y|=|g x-y|$. After change of variables $y=g y^{\prime}$ and application of irreducible representation matrices unitarity, we arrive at

$$
\sum_{i}^{s} T_{g} \hat{J}_{i} \Psi_{j}=\sum_{k, p}^{s} D_{j p}^{\nu}(g) \frac{\int \Psi_{k}^{*}(y) \Psi_{p}(y)}{|g(x-y)|} d y \Psi_{k}(x)=\sum_{k, p}^{s} D_{j p}^{\nu}(g) \hat{J}_{k} \Psi_{k}
$$

and on the Coulomb one

$$
\sum_{i}^{s} T_{g} \hat{C}_{i} \Psi_{j}=\sum_{k, p}^{s} D_{j k}^{\nu}(g) \int \frac{\Psi_{p}^{*}(y) \Psi_{p}(y)}{|g(x-y)|} d y \Psi_{k}=\sum_{k, p}^{s} \hat{C}_{p} D_{j k}^{\nu}(g) \Psi_{k} .
$$

An account of symmetry with respect to the permutation group is studied in [4], where a determinant representation for the eigenfunctions was introduced. It explored by Fock in derivation of one-particle self-consistent equations via variational principle in terms of wave functions from irreducible subspaces [2]. The space transformation group action may be determined also via transformation of arguments.

A general symmetry transformations of the system of equations (15) is determined as in Sec. 2.

An irreducible representation basis is fixed by the projection operators $P_{i k}^{\nu}$, numerated by indices $\nu, i k$ :

$$
P_{i k}^{\nu}=\sum_{G} N_{\nu} D_{i k}^{\nu}(g) T_{g}
$$

normalized by $N_{\nu}$.

The system of Fock equations then is rearranged under permutations action while the exchange integrals form the set $J_{g}$. The Coulombian and differential parts are invariant under permutations.

The eigenvalue problem of the exchange operators, numerated by the group elements $g$ after summation by symmetry group is solved via:

$$
\operatorname{det}\left[\sum_{g \in G} D_{i k}^{\nu}(g) J_{g}-\delta_{i k} E\right]=0
$$


[4] that we take as the initial point of further investigation.

\subsection{Heisenberg chain perturbation}

Stark effect. A perturbation theory by the field $\epsilon=e E$ directed along $x$ is applied to a solution $\phi$ of the perturbed Fockian:

reads as expansion by small parameter $\epsilon$ :

$$
H \phi=\left(H_{F}+\epsilon x\right) \phi
$$

$$
\phi=\phi_{0}+\epsilon \phi^{(1)}+\ldots
$$

In the first order it gives the following expression for an eigen state:

$$
\phi_{n}^{(1)}=\sum_{m}^{\prime} \frac{(m|x| n)}{E_{m}-E_{n}} \phi_{0 m}
$$

where $(m|x| n)$ are matrix elements of the Cartesian coordinate $x$ in nonperturbed states. Plugging (26) into exchange integrals $J_{i k}$, defined for the electrons coupled to the centers $i, k$, by the expression (21):

$$
J_{i k}=\frac{e^{2}}{2} \iint \frac{\rho_{i k}\left(\vec{r}, \vec{r}^{\prime}\right)}{\left|\vec{r}-\vec{r}^{\prime}\right|} d \vec{r} d \vec{r}^{\prime},
$$

where, in a spirit of Fock paper [10], it may include all interacting electrons of the closest neighbors, as:

$$
\begin{gathered}
\rho\left(\vec{r}, \vec{r}^{\prime}\right)=\sum_{p} \phi_{p}^{*}(\vec{r}) \phi_{p}\left(\vec{r}^{\prime}\right)= \\
\sum_{p}\left(\phi_{0 p}^{*}(\vec{r})+\epsilon \phi_{p}^{(1) *}(\vec{r})\right)\left(\phi_{0 p}\left(\vec{r}^{\prime}\right)+\epsilon \phi_{p}^{(1)}\left(\vec{r}^{\prime}\right)\right)= \\
\sum_{p} \phi_{0 p}^{*}(\vec{r}) \phi_{0 p}\left(\vec{r}^{\prime}\right)+\epsilon \sum_{p}\left[\phi_{p}^{(1) *}(\vec{r}) \phi_{0 p}\left(\vec{r}^{\prime}\right)+\phi_{0 p}^{*}(\vec{r}) \phi_{p}^{(1)}\left(\vec{r}^{\prime}\right)\right]+o\left(\epsilon^{2}\right)= \\
\rho_{0}\left(\vec{r}, \vec{r}^{\prime}\right)+\epsilon \rho_{1}\left(\vec{r}, \vec{r}^{\prime}\right)+\ldots
\end{gathered}
$$

In the case of Stark effect:

$$
J_{i k}=\frac{e^{2}}{2} \iint \frac{\rho_{0}\left(\vec{r}, \vec{r}^{\prime}\right)}{\left|\vec{r}-\vec{r}^{\prime}\right|} d \vec{r} d \vec{r}^{\prime}+\epsilon \frac{e^{2}}{2} \iint \frac{\rho_{1}\left(\vec{r}, \vec{r}^{\prime}\right)}{\left|\vec{r}-\vec{r}^{\prime}\right|} d \vec{r} d \vec{r}^{\prime}=J_{i k}^{0}+e E J_{i k}^{1},
$$

we obtain the perturbed Heisenberg chain spin Hamiltonian [11]:

$$
H=J_{i k}\left(\vec{S}_{i}, \vec{S}_{k}\right)=\left(J_{i k}^{0}+e E J_{i k}^{1}\right)\left(\vec{S}_{i}, \vec{S}_{k}\right)
$$

with obvious Ising case:

$$
\begin{gathered}
J_{i k}^{1}=\frac{e^{2}}{2} \iint \frac{\rho_{1}\left(\vec{r}, \vec{r}^{\prime}\right)}{\left|\vec{r}-\vec{r}^{\prime}\right|} d \vec{r} d \vec{r}^{\prime}= \\
\frac{e^{2}}{2} \iint \frac{\sum_{p}\left[\phi_{p}^{(1) *}(\vec{r}) \phi_{0 p}\left(\vec{r}^{\prime}\right)+\phi_{0 p}^{*}(\vec{r}) \phi_{p}^{(1)}\left(\vec{r}^{\prime}\right)\right]}{\left|\vec{r}-\vec{r}^{\prime}\right|} d \vec{r} d \vec{r}^{\prime} .
\end{gathered}
$$

Plugging (27) and using the shorthand $\iint=\int$ yields:

$$
\frac{e^{2}}{2} \int \frac{\sum_{p, m}^{\prime} \phi_{0 m}^{*}(\vec{r}) \frac{(n|x| m)}{E_{m}-E_{n}} \phi_{0 p}\left(\vec{r}^{\prime}\right)}{\left|\vec{r}-\vec{r}^{\prime}\right|} d \vec{r} d \vec{r}^{\prime}+\frac{e^{2}}{2} \int \frac{\left.\sum_{p, m}^{\prime} \phi_{0 p}^{*}(\vec{r}) \frac{(m|x| n)}{E_{m}-E_{n}} \phi_{0 m}\left(\vec{r}^{\prime}\right)\right]}{\left|\vec{r}-\vec{r}^{\prime}\right|} d \vec{r} d \vec{r}^{\prime},
$$

or, in a bit more compact from:

$$
\begin{gathered}
\frac{e^{2}}{2} \sum_{p, m}^{\prime} \frac{(n|x| m)}{E_{m}-E_{n}} \int \frac{\phi_{0 m}^{*}(\vec{r}) \phi_{0 p}\left(\vec{r}^{\prime}\right)}{\left|\vec{r}-\vec{r}^{\prime}\right|} d \vec{r} d \vec{r}^{\prime}+\frac{e^{2}}{2} \sum_{p, m}^{\prime} \frac{(m|x| n)}{E_{m}-E_{n}} \int \frac{\left.\phi_{0 p}^{*}(\vec{r}) \phi_{0 m}\left(\vec{r}^{\prime}\right)\right]}{\left|\vec{r}-\vec{r}^{\prime}\right|} d \vec{r} d \vec{r}^{\prime} \\
=\frac{e^{2}}{2} \sum_{p, m}^{\prime} \frac{(n|x| m)}{E_{m}-E_{n}} J_{m p}^{0}+\frac{e^{2}}{2} \sum_{p, m}^{\prime} \frac{(m|x| n)}{E_{m}-E_{n}} J_{p m}^{0},
\end{gathered}
$$

with the obvious notation for $J_{p m}^{0}$.

Having the results of evaluation of the Fockian eigenfunction, one may calculate (35), otherwise we consider $J_{i k}^{1}$ as a parameter. Similarly, the Zeeman effect may be included on basis of the perturbation of Hamiltonian by $V=\vec{\mu} \vec{B}, \vec{\mu}=\frac{\mu_{B}\left(g_{L} \vec{L}+g_{S} \vec{S}\right)}{\bar{h}}$. 


\section{The Heisenberg method of Gauss distribution parameter evaluation}

\subsection{On eigenvalues distribution}

The basic eigenvalue equation written for multi-electron spin system for a given symmetry group $g \in G$ reads $[1,4]$ :

$$
\operatorname{det}\left[\sum_{g \in G} D_{i k}^{\nu}(g) J_{g}-\delta_{i k} E\right]=0,
$$

where $\nu$ is an irreducible representation number, $J_{g}$ are exchange integrals. It defines the system of terms $\Gamma_{\nu}$ numerated by the irreducible representations of the symmetry group $G$, The Pauli principle yields: each value of the system spin corresponds to one system of terms:

$$
2 n=2+\ldots+2+1+\ldots+1 .
$$

The mean energy is expressed as the sum of the roots $\sum E_{i}$ of the Eq. (36) as a coefficient by $E^{n_{\nu}-1}$ :

$$
E^{\nu}=\frac{1}{n_{\nu}} \sum_{g} \chi^{\nu}(g) J_{g}
$$

here $\chi^{\nu}(g)=D_{i i}^{\nu}(g)$ are chartacters of the group. The next term in the polynomial by $E^{n_{\nu}-2}$ gives:

$$
\begin{gathered}
\sum_{n>m} E_{n} E_{m}=\sum_{i>k, g, g^{\prime}}\left[D_{i i}^{\nu}(g) D_{k k}^{\nu}\left(g^{\prime}\right)-D_{i k}^{\nu}(g) D_{k i}^{\nu}\left(g^{\prime}\right)\right] J_{g} J_{g^{\prime}}= \\
\frac{1}{2} \sum_{i, k, g, g^{\prime}}\left[D_{i i}^{\nu}(g) D_{k k}^{\nu}\left(g^{\prime}\right)-D_{i k}^{\nu}(g) D_{k i}^{\nu}\left(g^{\prime}\right)\right] J_{g} J_{g^{\prime}} .
\end{gathered}
$$

By the group property:

$$
D_{i k}^{\nu}(g) D_{k s}^{\nu}\left(g^{\prime}\right)=D_{i s}^{\nu}\left(g g^{\prime}\right)
$$

taking $D_{i i}^{\nu}(g)=\chi^{\nu}(g)$ into account:

$$
\sum_{n>m} E_{n} E_{m}=\frac{1}{2} \sum_{g, g^{\prime}}\left[\chi^{\nu}(g) \chi^{\nu}\left(g^{\prime}\right)-\chi^{\nu}\left(g g^{\prime}\right)\right] J_{g} J_{g^{\prime}} .
$$

Plugging $E_{n}=E^{\nu}+\Delta E_{n}$ yields:

$$
\begin{gathered}
\sum_{n>m} E_{n} E_{m}=\sum_{n>m}\left(E^{\nu}+\Delta E_{n}\right)\left(E^{\nu}+\Delta E_{m}\right)= \\
\frac{n_{\nu}\left(n_{\nu}-1\right)}{2}\left(E^{\nu}\right)^{2}+\sum_{n>m} \Delta E_{n} \Delta E_{m}
\end{gathered}
$$

As $\sum \Delta E_{n}=0$ :

$$
\sum_{n=1}\left(\Delta E_{n}\right)^{2}=-2 \sum_{n>m}\left(\Delta E_{n}\right)\left(\Delta E_{m}\right)
$$

From $(38,40,41,42)$ and $\chi^{\nu}(e)=n_{\nu}$ we derive:

$$
\sum_{n=1}\left(\Delta E_{n}\right)^{2}=\frac{-1}{n_{\nu}} \sum_{g, g^{\prime}}\left(\chi^{\nu}(g) \chi^{\nu}\left(g^{\prime}\right)-\chi^{\nu}\left(g g^{\prime}\right)\right) J_{g} J_{g^{\prime}} .
$$

Finally, the mean square deviation from the mean value $E^{\nu}$

$$
\overline{\left(\Delta E_{n}\right)^{2}}=\frac{1}{n_{\nu}^{2}} \sum_{g, g^{\prime}}\left(\chi^{\nu}\left(g g^{\prime}\right)-\chi^{\nu}(g) \chi^{\nu}\left(g^{\prime}\right)\right) J_{g} J_{g^{\prime}}
$$

The characters of the symmetry group are evaluated by Frobenius theorem application. In the simplest case of the symmetry group as direct product of the permutation group and space symmetry group, the irreducible representations are all possible direct products of the irreducible representations of the subgroups. The characters in this case are simple products of the correspondent characters. 


\subsection{Perturbations of mean eigenvalues}

The values of exchange integrals in external field (30) gives for (48):

$$
E^{\nu}=\frac{1}{n_{\nu}} \sum_{g} \chi^{\nu}(g)\left(J_{g}^{0}+e E J_{g}^{1}\right)=E_{0}^{\nu}+e E E_{1}^{\nu},
$$

which demonstrates the Stark effect for the mean energy for a given IR of the symmetry group.

The mean square deviation from the mean value $E^{\nu}$ turns now becomes:

$$
\begin{gathered}
\overline{\left(\Delta E_{n}\right)^{2}}=\frac{1}{n_{\nu}^{2}} \sum_{g, g^{\prime}}\left(\chi^{\nu}\left(g g^{\prime}\right)-\chi^{\nu}(g) \chi^{\nu}\left(g^{\prime}\right)\right)\left(J_{g}^{0}+e E J_{g}^{1}\right)\left(J_{g^{\prime}}^{0}+e E J_{g^{\prime}}^{1}\right) \approx \\
\frac{1}{n_{\nu}^{2}} \sum_{g, g^{\prime}}\left(\chi^{\nu}\left(g g^{\prime}\right)-\chi^{\nu}(g) \chi^{\nu}\left(g^{\prime}\right)\right) J_{g}^{0} J_{g^{\prime}}^{0}+ \\
\left.e E \frac{1}{n_{\nu}^{2}} \sum_{g, g^{\prime}}\left(\chi^{\nu}\left(g g^{\prime}\right)-\chi^{\nu}(g) \chi^{\nu}\right)\left(g^{\prime}\right)\right)\left(J_{g}^{0} J_{g^{\prime}}^{1}+J_{g^{\prime}}^{0} J_{g}^{1}\right)= \\
\frac{1}{n_{\nu}^{2}} \sum_{g, g^{\prime}}\left(\chi^{\nu}\left(g g^{\prime}\right)-\chi^{\nu}(g) \chi^{\nu}\left(g^{\prime}\right)\right) J_{g}^{0} J_{g^{\prime}}^{0}+2 e E \frac{1}{n_{\nu}^{2}} \sum_{g, g^{\prime}}\left(\chi^{\nu}\left(g g^{\prime}\right)-\chi^{\nu}(g) \chi^{\nu}\right)\left(g^{\prime}\right) J_{g}^{0} J_{g^{\prime}}^{1},
\end{gathered}
$$

after summation rearranging. The magnetic field is accounted for similarly.

\subsection{Permutation group characters}

The permutation group characters are listed in [4], the nonzero ones $(P=e, P \in(12),(123),(12)(34))$ are:

$$
\begin{gathered}
\chi^{\nu}(e)=n_{\nu}=\frac{(2 n) !(2 s+1)}{(n-s) !(n+s+1)}, \\
\chi^{\nu}(12)=\frac{(2 n-2) ! 2(2 s+1)}{(n-s) !(n+s+1)}\left(s^{2}+s+n^{2}-2 n\right), \\
\chi^{\nu}(123)=\chi^{\nu}(12)(34) .
\end{gathered}
$$

For a specific case the result depends on $J_{g}$ values. In a case of localized atomic states $J_{(12)}$ decays exponentially with distance. Therefore the main contribution arises from nearest neighbors. A number of the neighbors depends on atomic structure symmetry.

If to suppose that we account only the closest neighbors, taking for them $J_{g}=J$ and neglect by others the energy is:

$$
E^{\nu}=\frac{1}{n_{\nu}} \sum_{g} \chi^{\nu}(g) J_{g}=-z \frac{s^{2}+n^{2}}{2 n} J+J_{e}
$$

For calculation of $\overline{\left(\Delta E_{n}\right)^{2}}$, we need:

$$
A\left(g, g^{\prime}\right)=\frac{1}{n_{\nu}^{2}}\left(\chi_{\nu}(e) \chi^{\nu}\left(g g^{\prime}\right)-\chi^{\nu}(g) \chi^{\nu}\left(g^{\prime}\right)\right) J_{g} J_{g^{\prime}} .
$$

Taking only the highest powers of $n, s$ into account, we have:

$$
\begin{gathered}
1 . g=g^{\prime} \\
A_{(12)(12)}=\frac{\left(n^{2}-s^{2}\right)\left(3 n^{2}+s^{2}\right)}{4 n^{4}}, \\
\text { 2. g nad g' have one joint element } \\
A_{(12)(13)}=\frac{\left(n^{2}-s^{2}\right)\left(s^{2}\right)}{4 n^{4}}, \\
\text { 3. g nad g' have no joint element } \\
A_{(12)(34)}=\frac{\left(s^{2}-n^{2}\right)\left(s^{2}\right)}{2 n^{5}} .
\end{gathered}
$$

If the atom has $\mathrm{z}$ neighbors, the type 1. we meet $z n$ times, 2. - z(z-1) times and 3. - 3- $\frac{z^{2} n^{2}}{2}$ times. Finally by (42), a deviation from $E^{\nu}$ is $\Delta E_{\sigma} \approx J \sqrt{n}$.

$$
\overline{\left(\Delta E_{n}\right)^{2}}=J^{2} z \frac{\left(n^{2}-s^{2}\right)\left(3 n^{2}-s^{2}\right)}{4 n^{3}} .
$$




\subsection{Nanotube example}

The geometry of the nanoobject under consideration is shown at the Fig. 1, the positions of centers of adiabatic potentials, denoted in previous sections by $\vec{R}_{j}$ are marked by boldface points. The axis of space symmetry of the figure is $z$, that is used as rotations $R \in S$ axis. The translations $t_{a} \in S$ are performed along this axis. The transversal Cartesian coordinates are $x y$. Let the symmetry group $G$ be a direct product of space symmetry group $S$ and the group of permutation $\mathcal{P}, G=S \odot \mathcal{P}$.

The permutation group characters (the nonzero ones $P=e, P \in(12),(123),(12)(34)$ ) are listed in [4],

As it was mentioned, for localized states account, the main contribution arises from nearest neighbors arriving at (48) and (51). The number of the neighbors depends on the material atoms positions.

For the whole symmetry group that is a direct product $G=S \otimes \mathcal{P}, s \in S . P \in \mathcal{P}$, $\operatorname{dim} G=\operatorname{dim} \operatorname{Sim} \mathcal{P}$, the characters are also products

$$
\chi(g)=\chi(s) \chi(P)
$$

and mean energy for the term corresponding to the irreducible representation $\nu$ of the whole $G$, yields, by the general (48)

$$
E^{\nu}=\frac{1}{n_{\nu}} \sum_{g} \chi^{\mu}(s) \chi^{\nu_{1}}(P) J_{g} .
$$

The index $\nu$ is a pair $\mu, \nu_{1}$, where $\mu$ is the number of irreducible representation of the space group $S$, while $\nu_{1}-$ of the permutation group $\mathcal{P}$.

For the tube of atoms at $\vec{R}_{i}$, see the Fig. 1, the group is described as follows [12].

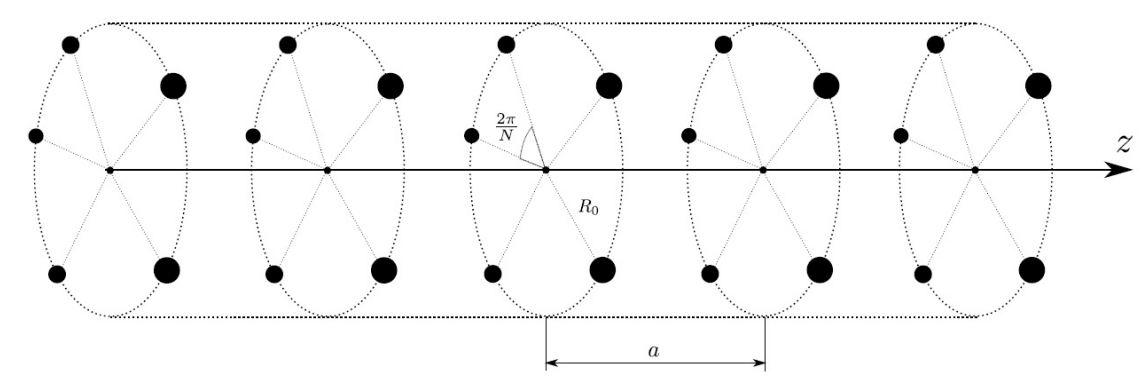

FIG. 1. The geometry of the tube centers positions

Let $A \in S$ be a group of the point-centers set symmetry and the finite group $R_{j} \in S_{c} \in S$ be a subgroup of rotations on angles $\phi_{j}=\frac{2 \pi j}{N}, j=0, \ldots, N-1$ in a plane, orthogonal to the $z$-axis. Let also the translations along $z$-axis are denoted as $t_{n a}, n=0, \ldots, M-1$ and form the discrete group $t_{n a} \in G_{T}$, hence the group elements are the pairs $A=\left(R_{j}, t_{n a}\right)$. The particular case relates to the structure of the tube is described by the direct product:

$$
S=S_{c} \otimes S_{T}
$$

Let the group is represented by transformations in the position space $\vec{r} \in R^{3}$. It is straightforward to see that $A$ is equivalent to the combined operations of rotation:

$$
R_{j}=\left(\begin{array}{ccc}
\cos \theta_{j} & \sin \theta_{j} & 0 \\
-\sin \theta_{j} & \cos \theta_{j} & 0 \\
0 & 0 & 1
\end{array}\right)
$$

and translation:

$$
t_{a}\left(\begin{array}{c}
x \\
y \\
z
\end{array}\right)=\left(\begin{array}{c}
x \\
y \\
z+a
\end{array}\right)
$$

The representation of the elements $A$ is written as:

$$
A \vec{r}=R_{j} \vec{r}+t_{a}^{n} \vec{r}=\left(\begin{array}{c}
\cos \theta_{j} x+\sin \theta_{j} y \\
-\sin \theta_{j} y+\cos \theta_{j} \\
z+n a
\end{array}\right) .
$$


The transformations form an Abelian group, $R_{j} t_{a}=t_{a} R_{j}$. In a realistic geometry of the tube and using periodic von Karman conditions we restrict the positive $n$ variable change so as $n \leq M$. The group element, or the transformation is parametrized by the vector $\vec{s}=\left\{s_{1}, s_{2}\right\}=\left\{\frac{2 \pi j}{N}, n a\right\}$. The transformation $A \in G$ is written as $A \vec{p}=\vec{p}+\vec{s}$.

For Abelian symmetry groups and cyclic subgroups, the IRs are one-dimensional. Conventionally choosing it as unitary, we write:

where

$$
D^{\vec{\mu}}(s)=\exp [i(\vec{\mu}, \vec{s})]
$$

$$
\vec{\mu}=\left\{\mu_{1}, \mu_{2}\right\}=\left\{j_{1}, j_{2}\right\},
$$

the integers, numerating irreducible representations run the values $j_{1}=1, \ldots, N ; j_{2}=1, \ldots, M$. Hence:

$$
D^{\vec{\mu}}(s)=\exp \left[i\left(j_{1} \frac{2 \pi j}{N}+\frac{2 \pi n}{M} j_{2}\right]\right.
$$

In such case, the sum that defines the mean energy is divided into two terms. One is "old", corresponding the unit element of the space group and the second relates to combined transformations.

Note that the dimension of the IR $n_{\nu}$ of $S \otimes \mathcal{P}$ is equal to dimension of the IR for the permutation group $\mathcal{P}$ because the dimension of it is unit:

$$
E^{\nu}=\frac{1}{n_{\nu}} \sum_{\vec{s}} \sum_{P} \exp [i(\vec{\mu}, \vec{s})] \chi^{\nu_{1}}(P) J_{P s} .
$$

The summation may be rearranged as:

$$
E^{\nu}=\frac{1}{n_{\nu}} \sum_{P} \chi^{\nu_{1}}(P) \sum_{\vec{s}} \exp [i(\vec{\mu}, \vec{s})] J_{P s} .
$$

In the model of equal exchange integrals the sum is proportional $\mathrm{J}$. The characters of space group do not contribute:

$$
\sum_{s} \chi^{\vec{\mu}}(s)=\sum_{j=0}^{N-1} \sum_{n=0}^{M-1} \exp \left[2 \pi i\left(j_{1} \frac{j}{N}+\frac{j_{2}}{M} n\right]\right.
$$

For nontrivial IR $\left(j_{1} \neq 0, j_{2} \neq 0\right)$ the sum (62) is the sum of the geometric progression, that gives:

$$
\sum_{j=0}^{N-1} \exp \left[2 \pi i\left(j_{1} \frac{j}{N}\right]=\frac{1-\exp \left[2 \pi i j_{1}\right]}{1-\exp \left[2 \pi i \frac{j_{1}}{N}\right]}=0\right.
$$

For the trivial IR $D^{0}(s)=1$, the sum is equal to $N M$. Generally, the sum in (61) depends on the model of $J_{P s}$ , that, for example, may depend on direction that link neighboring atoms. For the tube we study, the distance between neighbors along axis is $a$, while the neighbors at rings are at distance $b=2 R \sin \frac{\pi}{N}$. Generally $a \neq b$, hence we have two principal exchange integrals $J_{a}, J_{b}$. We divide the sum (58) into two terms:

$$
E^{\nu}=\frac{1}{n_{\nu}}\left[\sum_{P} \chi^{\nu_{1}}(P) \sum_{\vec{s}} \exp [i(\vec{\mu}, \vec{s})] J_{P s}+\sum_{P^{\prime}} \chi^{\nu_{1}}\left(P^{\prime}\right) \sum_{\vec{s}} \exp [i(\vec{\mu}, \vec{s})] J_{P^{\prime} s}\right] .
$$

The first contains permutations that are products of transpositions inside rings, while the second are built from transpositions along $z$ axis. Other permutations are not taken into account as related to non-closest neighbors.

We calculate the first internal sum by the space group:

$$
\begin{gathered}
\sum_{\vec{s}} \exp [i(\vec{\mu}, \vec{s})] J_{P s}=\sum_{j=0}^{N-1} \sum_{n=0}^{M-1} \exp \left[2 \pi i\left(j_{1} \frac{j}{N}+\frac{j_{2}}{M} n\right] J_{P s}=\right. \\
\sum_{n=0}^{M-1} \exp \left[2 \pi i \frac{j_{2}}{M} n\right] \sum_{j=0}^{N-1} \exp \left[2 \pi i j_{1} \frac{j}{N}\right] J_{b}
\end{gathered}
$$

For nontrivial IR we have zero, for trivial $M N J_{b}$. The second one is:

$$
\begin{gathered}
\sum_{\vec{s}} \exp [i(\vec{\mu}, \vec{s})] J_{P^{\prime} s}=\sum_{j=0}^{N-1} \sum_{n=0}^{M-1} \exp \left[2 \pi i\left(j_{1} \frac{j}{N}+\frac{j_{2}}{M} n\right] J_{P^{\prime} s}=\right. \\
\sum_{j=0}^{N-1} \exp \left[2 \pi i j_{1} \frac{j}{N}\right] \sum_{n=0}^{M-1} \exp \left[2 \pi i \frac{j_{2}}{M} n\right] J_{a}
\end{gathered}
$$


with the same result, but for $J_{a}$. The nonzero sum (66) for $\nu=\left\{0, \nu_{1}\right\}$ hence is equal to:

$$
E^{\nu}=\frac{M N}{n_{\nu}}\left[\sum_{P} \chi^{\nu_{1}}(P) J_{b}+\sum_{P^{\prime}} \chi^{\nu_{1}}\left(P^{\prime}\right) J_{a}\right] \text {. }
$$

The mean square deviation from the mean value $E^{\nu}, \nu=\left\{0, \nu_{1}\right\}$ is also splits as follows

$$
\overline{\left(\Delta E_{n}\right)^{2}}=\frac{1}{n_{\nu}^{2}} \sum_{g, g^{\prime}}\left(\chi^{\nu}\left(g g^{\prime}\right)-\chi^{\nu}(g) \chi^{\nu}\left(g^{\prime}\right)\right) J_{b}^{2}+\ldots
$$

in the first term the pair $g, g^{\prime}$ runs a ring set of atoms.

\section{Statistics}

\subsection{Magnetic field}

Let, following [1], the eigenvalues around the medium value are distributed by Gauss. As the number of terms, that belongs to a spin $s$ is equal to $n_{\nu}$, then, within the interval $E^{\nu}+\Delta E, E^{\nu}+\Delta E+d \Delta E$ one have for the number of terms

$$
\frac{n_{\nu}}{\sqrt{2 \pi \overline{\left(\Delta E_{n}\right)^{2}}}} \exp \left\{-\frac{\Delta E^{2}}{2 \overline{\left(\Delta E_{n}\right)^{2}}}\right\} d \Delta E .
$$

In the external magnetic field the additional energy of the system is:

$$
E^{\prime}=-\frac{e h}{2 \pi \mu} H m
$$

where $s \geq m \geq-s, \mu$ is electron mass.

The statistical sum of states:

$$
\begin{gathered}
\sum_{s=0}^{n} \sum_{m=-s}^{S} \int_{-\infty}^{\infty} d \Delta E \frac{n_{\nu}}{\sqrt{2 \pi \overline{\left(\Delta E_{n}\right)^{2}}}} \exp \left\{\alpha m+\beta \frac{s^{2}}{2 n}-\frac{\Delta E}{k T}-\frac{\Delta E^{2}}{2 \overline{\left(\Delta E_{n}\right)^{2}}}\right\}= \\
\sum_{s=0}^{n} \sum_{m=-s}^{s} n_{\nu} \exp \left\{\alpha m+\beta \frac{s^{2}}{2 n}+\frac{\overline{\Delta\left(E_{n}\right)^{2}}}{2 k^{2} T^{2}}\right\}
\end{gathered}
$$

where:

Plugging (51) gives:

$$
\begin{gathered}
\alpha=\frac{e h}{2 \pi \mu k T} H \\
\beta=\frac{z J}{k T}
\end{gathered}
$$

$$
Z=\sum_{s=0}^{n} \sum_{m=-s}^{s} n_{\nu} \exp \left\{\alpha m+\beta \frac{s^{2}}{2 n}+\beta^{2} \frac{s^{2}\left(4 n^{2}-s^{2}\right)}{8 n^{3} z}\right\}
$$

Denoting:

and taking into account:

$$
g(s)=\exp \left\{\frac{\beta s^{2}}{2 n}\right\}
$$

$$
n_{\nu}=\left(\begin{array}{c}
2 n \\
n+s
\end{array}\right)-\left(\begin{array}{c}
2 n \\
n+s+1
\end{array}\right)
$$

after reordering summation one obtains:

$$
Z=\sum_{m=-n}^{n} \sum_{s=|m|}^{n} \exp \{\alpha m\} g(m)\left[\left(\begin{array}{c}
2 n \\
n+s
\end{array}\right)-\left(\begin{array}{c}
2 n \\
n+s+1
\end{array}\right)\right] .
$$

After transformation similar to integrating by parts, we have:

$$
Z=F \sum_{m=-n}^{n} \exp \{\alpha m\} g(m)\left(\begin{array}{c}
2 n \\
n+m
\end{array}\right) .
$$


One may show that the expression $\exp \{\alpha m\} g(m)\left(\begin{array}{c}2 n \\ n+m\end{array}\right)$ has the sharp maximum at a point $m_{0}$, hence the Taylor expansion in the vicinity of this point may be a good approximation in the exponent of $g(m)$. Approximately:

$$
\begin{gathered}
g(m)=\exp \left\{\frac{\beta n_{0}^{2}}{2 n}+\beta \frac{m_{0}}{n}\left(m-m_{0}\right)-\frac{\beta^{2}}{8 n^{3} z}\left[4 m_{0}^{2} n^{2}-m_{0}^{4}+\right.\right. \\
\left.\left.8 m_{0} n^{2}\left(m-m_{0}\right)-4 m_{0}^{3}\left(m-m_{0}\right)\right]\right\},
\end{gathered}
$$

the statistical sum $Z$ takes the form:

$$
\begin{gathered}
Z=F \sum_{m=-n}^{n} \exp \left\{\left(\alpha+\beta \frac{m_{0}}{n}-\beta^{2} \frac{m_{0}^{3}}{2 n^{3} z}\right) m\right\} g(m)\left(\begin{array}{c}
2 n \\
n+m
\end{array}\right)= \\
F\left[2 \cosh \left(\frac{\alpha+\beta \frac{m_{0}}{n}-\beta^{2} \frac{m_{0}}{n z}+\beta^{2} \frac{m_{0}^{3}}{2 n^{3} z}}{2}\right)\right]^{2 n} .
\end{gathered}
$$

The space symmetry account is based on modifications of the characters formula in expressions for energy (66) and the Gauss distribution parameter (67).

\subsection{Electric field}

The electric field perturbs the exchange integral as it is prescribed by (30)

$$
\beta=\frac{z\left(J_{0}+\epsilon J_{1}\right)}{k T}=\beta_{0}+\epsilon \beta_{1} .
$$

Plugging it into (85) yields:

$$
\begin{gathered}
\frac{Z}{F}=\left[2 \operatorname { c o s h } \left(\frac{\left.\left.\alpha+\left(\beta_{0}+\epsilon \beta_{1}\right) \frac{m_{0}}{n}-\left(\beta_{0}+\epsilon \beta_{1}\right)^{2} \frac{m_{0}}{n z}+\left(\beta_{0}+\epsilon \beta_{1}\right)^{2} \frac{m_{0}^{3}}{2 n^{3} z}\right)\right]^{2 n}=}{2}=\right.\right. \\
{\left[2 \cosh \left(\frac{\alpha+\beta_{0} \frac{m_{0}}{n}-\beta_{0}^{2} \frac{m_{0}}{n z}+\beta_{0}^{2} \frac{m_{0}^{3}}{2 n^{3} z}+\epsilon \beta_{1} \frac{m_{0}}{n}\left(1-\frac{2}{z}+\frac{m_{0}^{2}}{2 n^{2} z}\right)+\ldots}{2}\right)\right]^{2 n},}
\end{gathered}
$$

the quadratic terms in $\epsilon$ are not shown.

\subsection{To thermodynamics}

The most probable value of magnetic moment is determined as:

$$
m_{0}=\frac{\partial \ln Z}{\partial \alpha}=n \tanh \frac{\alpha+\beta \frac{m_{0}}{n}-\beta^{2} \frac{m_{0}}{n z}+\beta^{2} \frac{m_{0}^{3}}{2 n^{3} z}}{2} .
$$

The neglected term with $\ln F$ is of order "1". Further expansion of $\frac{m_{0}}{n}$ in power series of $\epsilon$ gives the coefficients in the equation of state [13]:

$$
\left.\frac{\partial m_{0}}{n \partial \epsilon}\right|_{\epsilon=0}=\beta_{1}\left(\frac{1}{2}-\frac{1}{z}+\frac{m_{0}^{2}}{2 n^{2} z}\right)\left(1-\tanh ^{2} \frac{\alpha+\beta_{0} \frac{m_{0}}{n}-\beta_{0}^{2} \frac{m_{0}}{n z}+\beta_{0}^{2} \frac{m_{0}^{3}}{2 n^{3} z}}{2}\right) \frac{m_{0}}{n} .
$$

The derivative of $\ln Z$ by $\epsilon$ gives the polarization per atom:

$$
\frac{p_{0}}{n}=\frac{\partial \ln Z}{n \partial \epsilon}=\beta_{1} \frac{m_{0}}{n}\left(\frac{1}{2}-\frac{1}{z}+\frac{m_{0}^{2}}{2 n^{2} z}\right) \tanh \left(\frac{\alpha+\beta \frac{m_{0}}{n}-\beta^{2} \frac{m_{0}}{n z}+\beta^{2} \frac{m_{0}^{3}}{2 n^{3} z}}{2}\right),
$$

which demonstrates electromagnetic effects. The energy (66) and the Gauss distribution parameter (67) modifications for nanotubes naturally introduce the closest neighbors exchange integrals defined by the nano-object symmetry. This gives a model thermodynamic description on base of a model of statistical sum. 


\subsection{Discussion}

The expression (83) differs from Weiss formula by $\cot -1 / x \rightarrow \tanh x$ because of two spin orientation and cubic term. Set:

arriving at the equations:

$$
\frac{m_{0}}{n}=y, \quad \frac{\alpha+\beta \frac{m_{0}}{n}-\beta^{2} \frac{m_{0}}{n z}+\beta^{2} \frac{m_{0}^{3}}{2 n^{3} z}}{2}=x,
$$

$$
\text { I. } y=\tanh x
$$

$$
\text { II. } \quad \alpha+\beta y-\beta^{2} \frac{y}{z}+\beta^{2} \frac{y^{3}}{2 z}-2 x=0 .
$$

Plots Fig. 2, $\alpha=0.2, \eta=2, z=8$, ferromagnetism condition (II curve inclination at $y=0$ is less than one for I.)

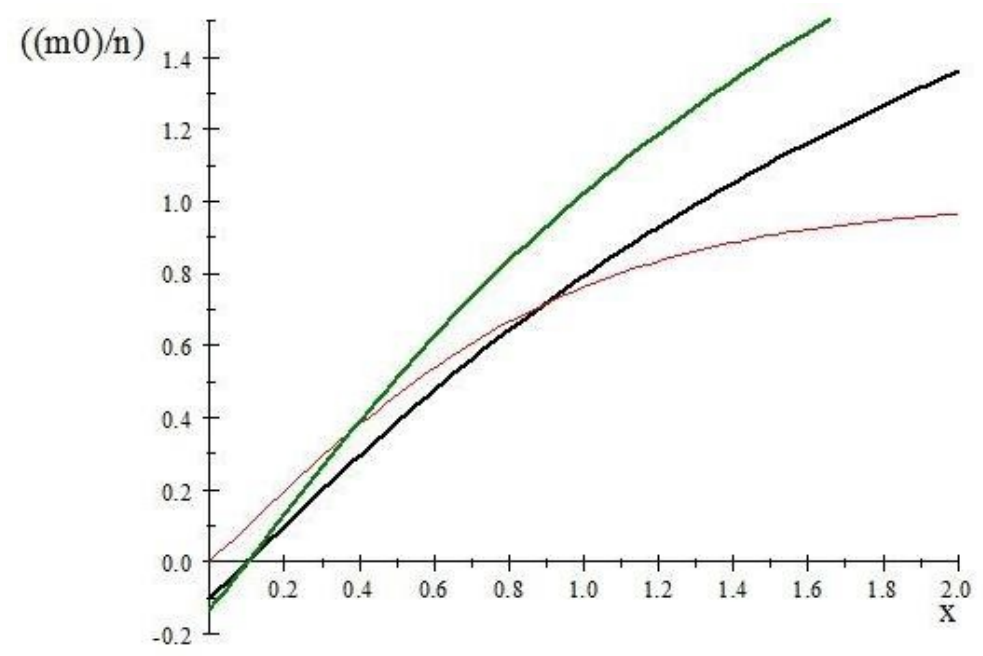

FIG. 2. Graphic solution of (85)

The next figure Fig. 3 presents number of closest neighbors as function $J / k T$.

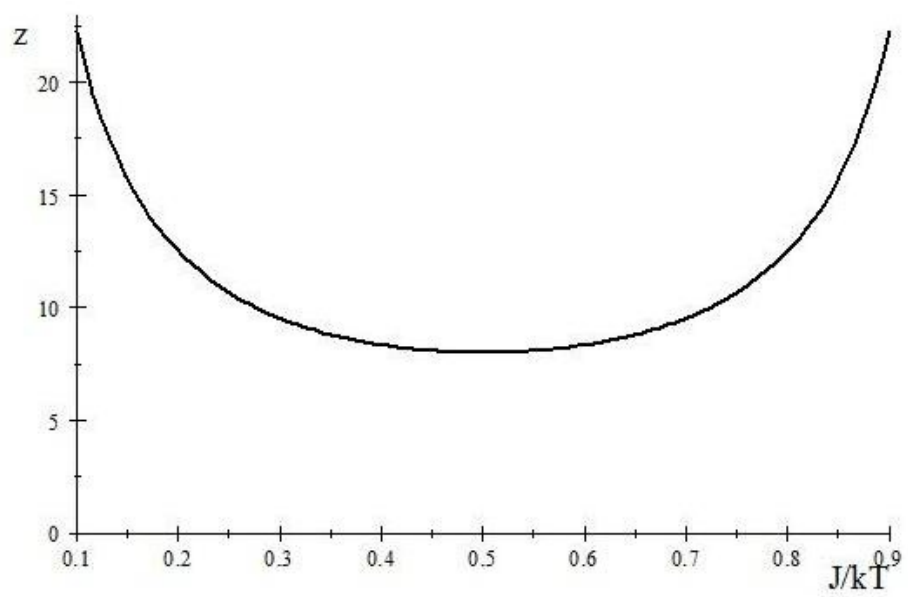

FIG. 3. The number of neighbors as function $\frac{J}{k T}$

$$
\beta\left(1-\frac{\beta}{z}\right) \geq 2
$$

maximum of the 1.h.s. is $\beta_{\max }=1 / 2$, that yields

$$
z \geq 8
$$


a good example is $z_{F e}=8$.

A small $\alpha$ allows the expansion $\left(t h x \approx x-x^{3} / 3+\ldots\right)$, that gives the following dependence on $\beta$

$$
y=\frac{\alpha}{2-\beta+\frac{\beta^{2}}{z}}+\frac{\alpha^{3}}{\left(2-\beta+\frac{\beta^{2}}{z}\right)^{4}}\left(\frac{\beta^{2}}{2 z}-\frac{2}{3}\right),
$$

The first term of the series gives the Weiss modification of Curie law

with the Curie point

$$
m_{0}=\frac{1}{T-\Theta} \frac{T}{T\left(1+\sqrt{1-\frac{8}{z}}\right)-\Theta\left(1-\sqrt{1-\frac{8}{z}}\right)}
$$

$$
\Theta=\frac{2 J}{k\left(1-\sqrt{1-\frac{8}{z}}\right)} .
$$

Within the concept suggested in this paper, the electric field account is introduced via its exchange integral perturbation by Stark effect (30).

\section{Conclusion}

The Zeeman energy we also can consider as a Zeeman effect, that add a perturbation to the exchange integral.

Both from microscopic (multipole expansion) and macroscopic (symmetry-based expansion of the free energy) points of view, with Dzialoshinski-Moryja interaction account see [14].The great interest to the magnetoelectric effects is expressed in abundant publications [15].

An equivalence of the nonlinear Schrödinger equation and the equation of a Heisenberg ferromagnet [16] points out possible applications of the advanced NS equation perturbation theory.

The important perspective of more deep understanding of the chemical bonds contains in the Fock paper [9]; let us put here a citation: “...idea of a bond which is built by a pair of electrons assumes such strong interaction between the electrons with opposite spins that any description of this interaction by means of two one-electron functions is somewhat artificial and cannot be exact. It is much more natural to associate with each saturated bond its own two-electron function". This also leads also to more natural notion and values of exchange integrals. The ideas of Heitler and Heisenberg are unified also with Floquet theory for non-localized eigenstates of Fock Hamiltonian and specified in the context of Wigner theorem [6].

\section{References}

[1] Heisenberg W. Zur Theorie des Ferromagnetismus. Zeitschrift für Physik, 1928, 49(9-10), P. 619-636.

[2] Fock V.A. An Approximate Method for Solving the Quantum Many-Body Problem. Reported at the Session of the Russian Phys.-Chem. Soc. on 17 December 1929. Selected works. Quantum Mechanics and Quantum Field Theory. Ed. by L.D. Faddeev, L.A. Khalfin, I.V. Komarov. P. 137-164, CRC Press, 2004.

[3] Popov I.Y., Melikhov I.F. The discrete spectrum of the multiparticle Hamiltonian in the framework of the Hartree-Fock approximation. Journal of Physics: Conference Series, 2014, 541. P. 012099/1-4.

[4] Heitler W. Zur Gruppentheorie der homopolaren chemischen Bindung. Zs. Phys., 1928, 47, P. 835-838.

[5] Geyler V.A., Popov I.Yu. Group-theoretical analysis of lattice Hamiltonians with a magnetic field. Phys. Lett. A., 1995, 201, P. 359-364.

[6] Wigner E. Group Theory. Academic Press, New York, London, 1959.

[7] Petrashen M., Trifonov E. Applications of Group Theory in Quantum Mechanics. Dover publication, 2009.

[8] Jens P. Dahl: Introduction to The Quantum World of Atoms and Molecules. World Scientific Publishing, Singapore, 2001.

[9] Fock V.A. Application of Two-Electron Functions in the Theory of Chemical Bonds. Selected works. Quantum Mechanics and Quantum Field Theory. Ed. by L.D. Faddeev, L.A. Khalfin, I.V. Komarov. P. 519-524, CRC Press, 2004.

[10] Fock V.A. On Quantum Exchange Energy. Selected works. Quantum Mechanics and Quantum Field Theory. Ed. by L.D. Faddeev, L.A. Khalfin, I.V. Komarov. P. 263-278, CRC Press, 2004.

[11] Roberts J.A.G., Tompson C.J. Dynamics of the classical Heisenberg spin chain. Journal of Physics A: Mathematical and General, 1988, 21(8), P. 1769-1780.

[12] Leble S. Cyclic-periodic ZRP structures. Scattering problem for generalized Bloch functions and conductivity. Nanosystems: physics, chemistry, mathematics, 2018, 9(2), P. 225-243.

[13] Dzyaloshinskii I.E. On the magneto-electrical effect in antiferromagnets. J. Exp. Theor. Phys. (U.S.S.R.), 1959, 37, P. 881-882.

[14] Chandrashekar Radhakrishnan, Manikandan Parthasarathy, Segar Jambulingam and Tim Byrnes. Quantum coherence of the Heisenberg spin models with Dzyaloshinsky-Moriya interactions. Scientific Reports, 2017, 7, P. 13865.

[15] Spaldin, Nicola A., Fiebig, Manfred. The renaissance of magnetoelectric multiferroics. Science, 2005, 309(5733), P. 391-392.

[16] Zakharov V.E., Takhtajan L.A. Equivalence of the nonlinear Schrödinger equation and the equation of a Heisenberg ferromagnet. Theor. Math. Phys., 1979, 38, P. 17-23.

[17] Heitler W. Stäorungsenergie und Austausch beim Mehrkäorperproblem. Zs. Phys., 1927, 46, P. 47. 